\title{
New Technological Trends in Healthcare
}

\author{
Serik Smagulov, \\ Viktoriya Smagulova MD
}

\begin{abstract}
This article addresses the question of what today means improving the quality of medical care and reducing the costs associated with introducing innovations in healthcare. Researchers must understand the development of technological trends of the health care system, that will lead to improve the quality of services and, consequently, the quality of life. We consider various fields of activity, starting with Big Data, in the field of medicine and pharmacology, and ending with robotics and chip making. The result is reduced costs and improved quality of care and patient care.

Keywords: Digital transformation, digitization, big data, robotics, chips, pharmaceutical companies, the future of medicine.
\end{abstract}

In our time, we are witnessing a digital transformation that is rapidly taking place in the field of healthcare. The focus is on exemplary experience in patient care, and pharmaceutical companies and clinical care centers have made radical changes through technological innovation and communication.

Technology has become almost the primary way to increase life expectancy. Technology can save lives. And this has been proven more than once. Fortunately, the scope of techniques for saving lives is greatly expanded.

What does digital transformation mean for the healthcare industry?

Digital transformation is a trend that can only be realized when organizations can rethink business processes. Simply put, digital transformation in healthcare is the use of various forms of digital technology that help the patient lead a better life. The need of the patient becomes the center of the business.

Medical organizations can be intimidated by the enormous amounts of data that they will have to deal with because the data passing through their systems is often unstructured and difficult to manage.

Digital transformation involves various areas of activity in the health care system, ranging from patient registration to smart diagnosis [1]. 
Consider some of them.

- Big Data used in medicine.

For the first time, the term "Big Data" (Big Data) was used in 2008 by the British journal «Nature» which devoted a special issue to the phenomenon of explosive growth in the volume and diversity of data processed. Big Data analysis has become a significant boon for both patient management and the business aspect of health organizations.

The main strategies for applying Big Data in medicine are the creation of medical data registers in which information can be exchanged, the use of accumulated knowledge to predict possible "waves" of diseases, and the introduction of electronic cards for the patient that will be available to every doctor who treats him [2]. Analysis of various data allows for a detailed and comparative study of the patient, to find out his lifestyle and the lifestyle of similar patients, to develop the right treatment strategy.

Big Data can collect all the data and information about what constitutes a disease from DNA, proteins, and metabolites to organs, organisms, cells, tissues and ecosystems [3]. In this way, they can help build better health profiles and prognostic models around patients to diagnose better and treat diseases.

There are more advantages of using Big Data in medicine. For example, the Toronto Children's Hospital has implemented Project Artemis. The hospital information system collects and analyzes data on infants in real time. The system can track every 1260 indicators of the state of each child, allows you to predict the unstable state, and in time to start the prevention of diseases in children.

Big data and artificial intelligence are now widely used in pharmaceutical manufacturing and marketing of drugs. The most significant effect of Big Data is expected in the modeling of new drugs. An example of work in this direction is the activities of the IT company Semantic Hub, which is developing services to assess the prospects for the development of new products.

About the collection of clinical data about patients, pharmaceutical companies are seeking access to medical data of patients and racing deals with technology companies with experience in the analysis of Big Data. 
Big Data helps to improve the quality of clinical trials. Using these technologies, companies can make clinical trials more efficient. From several databases, analytical systems can select patients who best meet the preliminary requirements of the drug test.

It is imperative to identify the side effects of drugs timely. Big Data is widely used in this area. It is very important that they are used in the prediction of side effects for specific compounds and components even before the start of clinical trials.

Using an analytical method that includes checking dozens of different drug characteristics, companies can save time, money and save patients' lives.

- Medical robotics.

Recently, robotics is continuously being introduced into the healthcare industry. Medical robots are the result of interdisciplinary efforts to automate healthcare. There are robots designed to facilitate the work of nurses, for example, robots for injection and collection of tests, trolley robots to bypass patients who can enter data in the history based on the doctor's speech. Although robots have been part of the health care system for several decades, from small laboratory models to complex ones that can either perform operations on their own or help a human surgeon, the possibilities of using them in medicine have only recently expanded. Robots are currently used to help people with sensory, cognitive and motor impairments [4].

Advances in technology and computers have helped faster in sequencing genomes and a cost-effective manner. This helped scientists to understand in detail the genetic profile of a person and thereby prescribe treatment methods that can lead to more effective treatment.

Doctors, clinicians, and specialists use genomic sequencing for more accurate diagnosis and more precise treatment of their patients. The increase in technology and computing power allows scientists to create a fully personalized form of testing and treatment.

- The use of chips.

The biochip is the result of the merger of two areas of knowledge - biotechnology and semiconductor technology. Source - Biochip in a compact format allows you to identify various intracellular processes, analyzing hundreds or thousands of reactions at the same time. 
One of the first commercial chips created the company Affymetrix - GeneChip. This chip was produced using microlithography technology. Biochips are organized placements of DNA molecules on a special carrier.

The use of proven methods of the electronics industry has led to impressive results.

Recently, there are examples of using electronic sensors to measure various types of treatment at the cellular level. As a result, the best possible treatment will be selected, and then assigned to the patient. This trend has been made possible by advances in DNA sequencing and through in-depth stem cell research [5].

There are precedents for the creation of chips of various organs, such as the kidney, heart, intestines, bone marrow, and so on. The organs on the chip device can supply cells with nutrients and oxygen, just as the cells of the human body are supported by blood. These cells grow and react just like real cells of a human organ.

Summing up, it can be noted that digital transformation is always a complicated process, especially if we consider such a complex industry as healthcare. Often you can meet some problems that may interfere with the process of digital transformations in health care. For the transformations to be successful, the industry as a whole must be ready for the change. For some health institutions, return on investment is in the first place, which in most cases puts digital transformation projects into a dead end. In our opinion, in the first place should still be patient care. And, as we see, this is what modern medicine seeks.

\section{References}

1. Yichuan Wang, LeeAnn Kung, Terry Anthony Byrd. Big data analytics: Understanding its capabilities and potential benefits for healthcare organizations // Technological Forecasting and Social Change. Volume 126, January 2018, Pages 3-13.

2. Dhindsa K. What's holding up the big data revolution in healthcare? BMJ 2018; 363 doi: https://doi.org/10.1136/bmj.k5357 (Published 28 December 2018).

3. Mohammad Adibuzzaman, Poching DeLaurentis, Jennifer Hill, Brian D. Benneyworth. Big data in healthcare - the promises, challenges, and opportunities from a research perspective: A case study with a model database // AMIA Annu Symp Proc. 2017; 2017: 384-392. Published online 2018 Apr 16. 
4. Medical Robotics. Edited by Vanja Bozovic. Published: January 1st 2008. DOI: $10.5772 / 54929$.

5. Akio Okia, Hiroki Ogawaa, Masao Nagaia, Satomi Shinbashia, Madoka Takaic, Akinori Yokogawab, Yasuhiro Horiikea. Development of healthcare chips checking life-stylerelated diseases. // Materials Science and Engineering: C. Volume 24, Issues 6-8, 1 December 2004, Pages 837-843. 